\title{
Hypoxia: Modern Ideas of the Action Mechanism, System and Behavioral Reactions
}

\author{
E.J. Mehbalieva*
}

Doctor of Sciences in Biology, Azerbaijan National Academy of Sciences, Azerbaijan Republic

*Corresponding author

\section{A B S T R A C T}

\section{Keywords \\ Hypoxia, Primary and secondary changes, Functional- systemic reactions, Behavioral acts \\ Article Info \\ Accepted: \\ 12 March 2019 \\ Available Online: 10 April 2019}

\section{Introduction}

In evolution the vast majority of animal organisms adopting oxygen gas $\left(\mathrm{O}_{2}\right)$ from the environment has gained a great bioenergetic advantage also aerobic respiration style and the acquisition of mechanisms for life activities and adaptations [4]. Aerobic organisms can only survive in oxygen environments and they oxidize nutrients through molecular oxygen, convert potential chemical energy into new free bioenergy form (mainly ATP). This process takes place in all the cells of the body, in their mitochondrial particles (in the "energy blocks" "power aparats" of cells). The evolution also has been preserved in higher aerobic organisms, also in the old-tested limited bioenergetic mechanism- ATP- produced without oxygen(anerobic) (for example, glycolysis cycle).

According to calculations, many animal organisms living on the land, which have well-functioning external respiratory tract and internal respiratory mechanisms they can use only $2 / 3$ of the oxygen gas, which is less than $21 \%$ in the air that are spends to the breathing of useful tissue (or cell). Oxygen transported into the organism is dispersed unevenly between tissue and organs: proper adaptive nerve and hemodynamic regulation 
mechanisms are constructed and operated that, some organs get less blood (oxygen), and some organs much. It depends on their general and specific activities and the nervous-humoral regulatory system. Head, brain, functional skeletal muscles and other organs require more oxygen [7].

As mentioned above, oxygen needs to the cells of the organism for the satisfy of great demand of free bioenergy (ATP). All the biosynthesis processes in the organism (synthesis of proteins, etc.) are possible at the expense of ATP and distributed relative standard as a result of hydrolysis of other macroheric molecular compounds is possible at the expense of spends free energy. For example, a large amount of ATP produced in nerve cells (neurons) is spent to the active activities of active wave propagation of nerve fibers-- transmembrane ion channels $(\mathrm{Na},-\mathrm{K}$, $\mathrm{Ca}$ and $\mathrm{Mg}$ "pumps"). For the contraction of muscle and movement activity are also needed large volumes of bioenergy. In animal cells $\mathrm{O}_{2}$ is also needed for the processes of formation of oxygen radicals, which in low concentration are affects to the intracellular metabolism positive, in high concentrations destructive: the latter state creates "oxidative stress" in the cell. Wellknown researcher in the field of bioenergetics V.P.Skulachev (1998) noted that oxygen in living cells is both a good and harmful chemical factor. Oxygen radicals can affect like a poison.

The presence of excess and low oxygen in the organism can cause suddenly damaging physical-chemical and molecular changes in its cells. In general, human or animal organisms without oxygen can live actively a very short time (maximum 3-5 minutes).

Hypoxia(synonyms: anoxia oxygen deficiency, oxygen starvation) radically is differs from the other damaging factors found in earth such as natural and artificial, acute, exo- or endogenous origin, at the same time characterized by large-scale early (primary) and delayed (secondary) reactions that are intuitively and explicitly frequently manifested and as a powerful factor which causes pathologies. Hypoxia is also a factor that draws attention to, clearly shows the most important metabolic processes and physiological functions in the body are interconnected with each other, their balance, durability, compensation and adaptive capabilities in case of hypoxia. So that, in despite the very distinctive effect range of hypoxia those criteria can be varied in the hierarchical plan at the most different levels with similar sequences. This can be regarded as a specific form of influence selected and manifested by its identity which was observed in a number of studies related to hypoxia.

About causes and forms of hypoxia in organism currently, there are richness of notion[5]. It is shown that, according to their nature, hypoxia can be exogenous and endogenous. In the first case, the initial reason is the amount of low oxygen content in the air $\left(\mathrm{PO}_{2}\right)$ absorbed from the environment during respiration(hypoxic hypoxia). In the second case, causes hypoxia is diffusion of oxygen into the blood in the lungs, transport of oxygen with hematolobine, aerohemotic barrier, infringement of the cellular (mitochondrial) respiratory tract, inhibition of synthesis and activation of respiratory enzymes (citroxrome enzymes) and other endogenous factors. As a result, may form respiratory, anemic, hemic, circulatory, mixed, histotoxic, acute, chronic and other hypoxic forms (variants). According to the generally accepted hypothesis, as a whole in the organism hypoxia leads to very complex changes and irregularities at the same time, organic contraindications cover a wide range, from molecular and metabolism level to the behavior covers a wide spectrum of 
biochemical and physiological parameters. The type of hypoxia and individual sensitivity, resistance and adaptation issues are also very complicated. We have already noted that, detailed learning and theoretical aspects in the problem of hypoxia are still stays[1].

We have tried to compile a common scheme of consistent results about effect of hypoxia on the based of a wide range of literature materials. Some of ideas about this have been put forward at the our previous studies [9].

The issues of systemic hypoxic effects are set as a topical issue in fundamental theoretical works of some authors $[7,11,12,16]$. This aspect can form a broader and more accurate scientific imagination about affecting mechanism of hypoxia. It is assumed that the systemicization of hypoxic reactions or effects should start from the level of the physical-chemical and molecular-cell. In this case, the introduction of hypoxic effects should be logical and serial (scanty) dependencies, development line, clearly visible order rule. As important initial hypoxic reactions on the level of various animal cells, especially on brain cells (neurons), are follows: Increased $\mathrm{pH}$, AMP and ADP, unused metabolites, decrease of components of oxidation-recovery system(NAD-H-NAD·P), hyper change of homeostasis, inactivation of mitochondrial enzymes and reduction of ATF synthesis, strengthening of glycolysis, inhibition of cytosol and membrane enzymes, disintegration of main metabolic ways, reduction of specific proteins, peptides, neuromediators, weakening of membrane ATP activity, beginning of the changes microstructure in synaptic membranesAppears secondary posthipoxic reactions in levels of tissues, including in the levels of brain tissue. To them belongs; tissue trophic and homeostasis disorder, Increased lysis (apoptosis) cases among cells, swelling of synaptic buds and and its decline, weakening of molecular receptor mechanisms, sharp change of membrane conductivity.

At the systems and at the behavioral level, during hypoxia among of saliently expressed reactions can be shown following; the intensification of delta rhythms of low frequency waves in the EEG spectrum of brain, fixeing hyper-awakening in the cortex and under cortex structures, infringement of the reflector activity, mnestic and cognitive discomfort, behavioral instability, acute and persistent reactions of different character in the emotional sphere, partial increase of vegetative dysfunction, changes in the general homeostasis, hormonal balance and immune system, breathless, sudden delay and activation cases in the movable (locomotor) acts, inadequate mental reactions, strengthening of adaptive reactions $[6,7,9$, $10,13,15$ and oth.].

The scientific ideas presented uptodate about influence more concrete mechanism of hypoxia on human or animal organisms shows that, as a whole at the root of hypoxic syndrome stays primarily in the bioenergetics of the cell - production macroergic ATP in the mitochondria, in the metabolic cycle disorders caused by oxygen deficiencyAccording to the theory of "Mitochondrial shock" [8], extensiveness and development of most pathogenetic properties and symptoms of hypoxia in any morphofunctional system it is closely related to the synthesis of energy (ATP) and the and violations of costs.

Some researchers working on the problem of hypoxia believe that, conducting experimental studies on functional systems and behavioral levels can give significant results. In this case, the issue of determining the major "markers" 
of the hypoxic effect is even more urgent[12]. Experimental works devoted to the study of blood and endocrine systems during acute and chronic hypoxia, movement and food behaviors, reactions in young animals for endurance and adaptation can be great interest by this regards. Some of these works have already been published [2, 3, 4 and oth.]. The main obtained results and their sequence are summarized below.

Scheme.1 System and behavioral reactions of mouse and rats in 1,2 and 3 months subjected to the various forms of experimental hypoxia

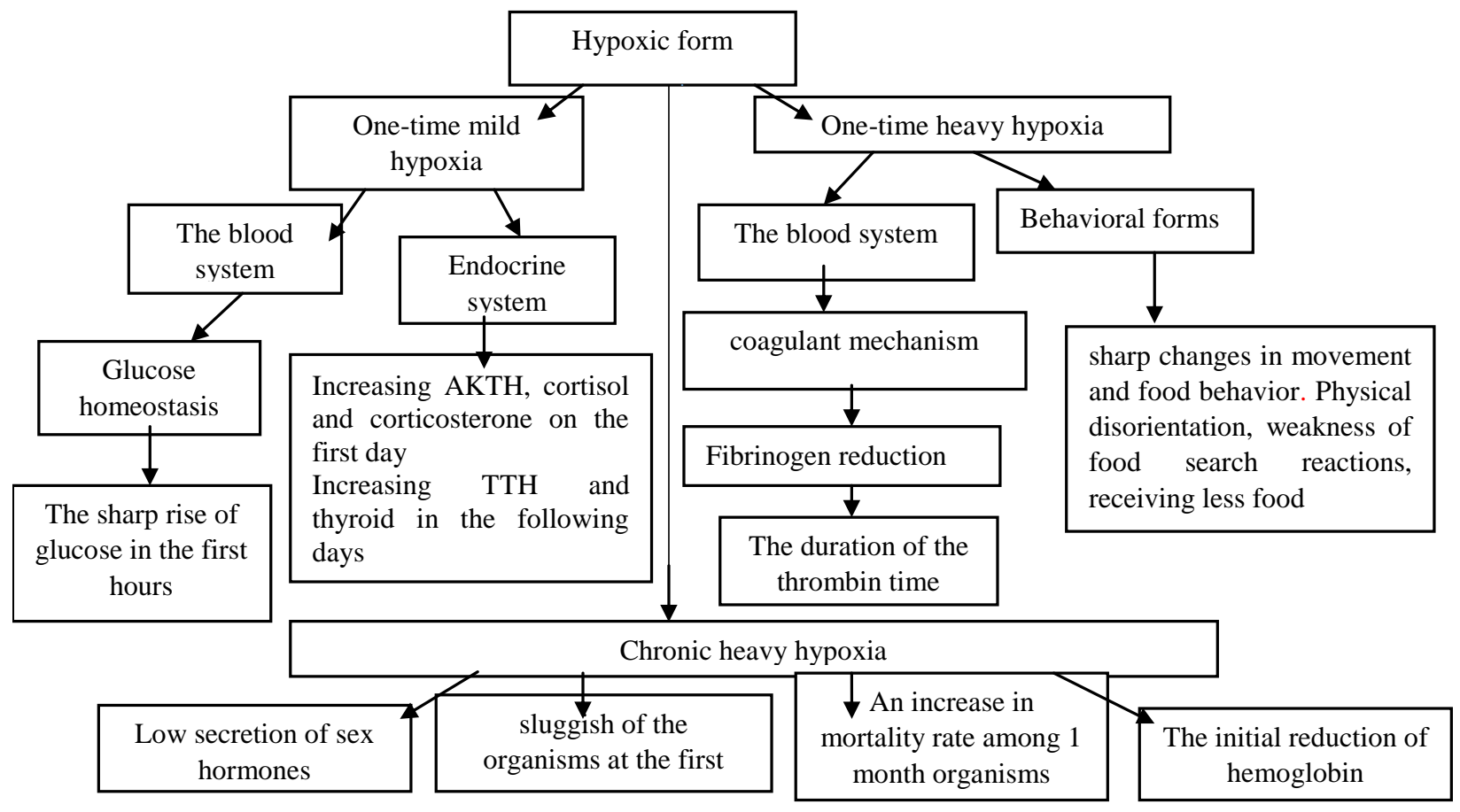

One of the most interesting facts revealed during our research is different features of hormonal reactions of pituitary-adrenocortical and pituitary-thyroid functional systems. Hypophysical trop hormone - AKTH and adrenocortical hormones - corticosterone and cortisol increase sharply in the beginning of hypoxia, then happens tendency to decrease, reminds reaction that happens immediate in the system during stress. Hypophysical trop hormone - TTH and thyroid thyroxine hormone $\left(T_{4}\right)$ is starts to rise relatively late during hypoxic effect.

Finally, it is important to note that, the main effects revealed on the general impact of hypoxia, their development character, physiological and pathophysiological evaluation issues problem is still actual, even though it is currently being resolved in a great deal and in this areas are needs for new original scientific researches and imaginations.

\section{References}

1. Mekhbalieva, E.J. 2013. The Problem of hypoxia: Fundamental aspects and the pathways of their experimental investigation // News of NAS of Azerbaijan, Ser.biol. med., Баки, 109116.

2. Mekhbalieva, E.J. 2015. Early reactions of the pituitary- 
adrenocortical system in rats under experimental hypoxia. Tr. Institute of Physiology of NAS of Azerbaijan, 33:107-113.

3. Mekhbalieva, E.J. 2016. Investigation of movement activity of young rats with oxygen deficiency// Scientific publications of the Institute of Physiology named after A.İ.Garayev of ANAS and Azerbaijan Society of Physiologists, Baku, Science, 34:7177.

4. Broda, E. 1978. Evolution of bioenergetic processes. M. World, 254.

5. Jukova, T.P., Palat Kh., Mochalova L.D. 1984. Causes of hypoxia / In the book Perinatal Pathology, under. ed. M.Y. Studenikina. M., Medicine, 4347.

6. Zakharov, E.M., Svinov M.M., Germanova E.L. and oth. 2004 Mechanisms of involvement of cholinergic systems in the processes of morphofunctional reorganization of the neocortex and hippocampus under hypoxia/Collection: "Problems of hypoxia: molecular, physiological and medical aspects, M., Origins, 268-296.

7. Kolchev, A.İ., Korovin A.B. 2000. Hypoxia of organs and systems. In the book: Hypoxia: adaptation pathogenesis, clinic. M., Medicine, 189-214.

8. Lukyanov, L.D. 1998. Mitochondrial dysfunction-typical pathological process, the molecular mechanism of hypoxia. In the book: Problems of hypoxia: molecular, physiological and medical aspects. M., Origins, 8-17.

9. Mekhbalieva, E.J. 2014. Primary and secondary reactions of nervous tissue to oxygen deficiency and their reflection in complex systemic functions. Bulletin of the Moscow State Regional University, 4:32-41.

10. Novikov, V.S., Shanin V.Y., Kozlov K.A. 2000. Hypoxia as a typical pathological process, its systematization / In the book: Hypoxia: adaptation, pathogenesis, clinic, M., Medicine, 12-22.

11. Skulchev, V.P. 1998. Oxygen in a living cell: good and evil. Soros Educational Journal, 3:2-10.

12. Soroko, S.İ., Burikh E.İ. 2004. Internal and intersystem rearrangements of physiological parameters during acute experimental hypoxia. Human Physiology, 30, 2: 58-66.

13. Buralda, B., Nyakas C., Vosselman H. Luiten P. 1995. Effects of early postnatal anoxia on adult learning and emotion in rats // Behav. Brain Res., 67: 85-90.

14. Mehbalieva, E.J. 2016. Reactions of same endocrine glands in male infant rats to acute hypoxia at the second stage of the sexual maturation. Ciencia e Tecnica Vitivincola, 31, 4: 193199.

15. Mikati, M., Zeinieh M., Kurdi R. et al. 2009. Longterm effects of acute and of chronic hypoxia on behavior. Brain Res., 157: 98-102.

\section{How to cite this article:}

Mehbalieva, E.J. 2019. Hypoxia: Modern Ideas of the Action Mechanism, System and Bchavioual Reactions. Int.J.Curr.Microbiol.App.Sci. 8(04): 1634-1638. doi: https://doi.org/10.20546/ijcmas.2019.804.190 